\title{
Adult-born neurons maintain hippocampal cholinergic inputs and support working
}

\section{memory during aging}

\section{Greer S. Kirshenbaum ${ }^{1,2}$, Victoria K. Robson ${ }^{1,2}$, Rebecca M. Shansky ${ }^{1,2,3}$, Lisa M. Savage ${ }^{4}$, E. David Leonardo ${ }^{1,2}$ and Alex Dranovsky ${ }^{1,2}$}

\author{
${ }^{1}$ Deparment of Psychiatry, Columbia University, New York, NY, 10032, USA \\ ${ }^{2}$ New York State Psychiatric Institute, New York, NY, 10032, USA \\ ${ }^{3}$ Currently in the Department of Psychology, Northeastern University, Boston, MA, 02115, USA \\ ${ }^{4}$ Department of Psychology, Binghamton University, Binghamton, NY, 13902, USA
}

\section{Contact}

Correspondence and requests for materials should be addressed to Alex Dranovsky (ad722@cumc.columbia.edu) or E. David Leonardo (el367@cumc.columbia.edu). 


\section{Summary}

Adult neurogenesis is impaired in disorders of stress, memory, and cognition though its normal function remains unclear. Moreover, a systems level understanding of how a small number of young hippocampal neurons could dramatically influence brain function is lacking. We examined whether adult neurogenesis sustains hippocampal connections across the life span. Long-term suppression of neurogenesis as occurs during stress and aging resulted in a progressing decline in hippocampal acetylcholine and the slow emergence of profound working memory deficits. These deficits were accompanied by compensatory rewiring of cholinergic dentate gyrus inputs such that ventrally projecting neurons were recruited by the dorsal projection. Our study demonstrates that hippocampal neurogenesis supports memory by maintaining the septohippocampal circuit across the lifespan. It also provides a systems level explanation for the progressive nature of memory deterioration during normal and pathological aging and indicates that the brain connectome is malleable by experience. 


\section{Introduction}

Connectivity between brain systems is thought to be established during developmental critical periods and then remain relatively fixed in adults. However, neurogenesis persists in the hippocampus throughout life, raising a possibility that the addition of new cells could facilitate systems-level circuit rewiring over time. This is especially intriguing since adult neurogenesis is highly susceptible to the effects of environmental changes and aging (Knoth et al., 2010; Kuhn et al., 1996; Spalding et al., 2013) presenting a putative mechanism for a very slow form of experience-based circuit plasticity in the adult brain. Stress and aging-induced reductions in neurogenesis correlate with decreased cognitive (van Praag et al., 2005) and emotional flexibility (Snyder et al., 2011). However, little is known about how ongoing neurogenesis influences hippocampal circuitry and function over time.

The role of young hippocampal neurons has been closely examined. Several studies have elucidated the dynamic properties of new hippocampal neurons throughout their maturation, and others have illuminated how new neurons integrate into existing brain circuits (Zhao et al., 2008). Young, 4-12 week old, hippocampal neurons have been found to play a role in the more difficult versions of hippocampal dependent cognitive tasks (Clelland et al., 2009; Shors, 2008) and a subtle, but significant role in stress regulation (Snyder et al., 2011). These findings emerged from short-term experimental reductions or increases in neurogenesis in rodents and together indicate a modest, but significant contribution of young neurons to hippocampal function. However, neurogenesis is an ongoing process, raising the possibility that long-term changes in the addition of new neurons to existing circuits could result in system-wide changes with more dramatic consequences for hippocampal function and for behavior. This notion is especially intriguing since it entertains time scales that reflect aging and the chronicity of illnesses where neurogenesis and hippocampal function are impaired. We therefore hypothesized that long periods of reduced neurogenesis, as observed in aging and chronic 
stress, would influence hippocampal connectivity with other brain systems. Since new neurons are added throughout life, we expected that long periods of reduced neurogenesis could lead to substantial connectivity changes.

We investigated whether long term reductions in hippocampal neurogenesis influences inputs into the hilus where hippocampal neurogenesis occurs. We reduced neurogenesis for 5 months in adult mice and observed a slowly progressing reduction of hippocampal acetylcholine and impairments in working memory. These changes corresponded to a significant remodeling of the cholinergic septohippocampal projection with recruitment of ventrally projecting neurons for innervation of both the dorsal and ventral hilus.

\section{Results}

\section{Long-term suppression of neurogenesis induces rewiring of cholinergic hilar inputs.}

We hypothesized that over time, reduction in neurogenesis would influence connectivity to the hilus, a structure where hippocampal afferents from many brain regions terminate to regulate neurogenesis, granule cell activity, and hippocampal function (Amaral et al., 2007). To model a reduction in hippocampal neurogenesis across aging, 2 month-old mice were treated with focal hippocampal X-irradiation (Santarelli et al., 2003) thereby dramatically and permanently reducing hippocampal neurogenesis (Figure S1). This method is thought to acutely target proliferating cells and also interfere with neural differentiation in the subgranular zone without dramatically influencing hippocampal and extra-hippocampal structures (Santarelli et al., 2003). We then used recombinant canine adenovirus (CAV), which undergoes selective retrograde neuronal transport (Kissa et al., 2002), to assess changes in hippocampal inputs over time. We injected CAV encoding green fluorescent protein (CAV-GFP) into the dorsal hilus of NG+ (Sham-irradiated mice with normal neurogenesis) and NG- (X-irradiated mice with diminished neurogenesis) animals after 5 or 2 months of reduced neurogenesis (Figure 1A). Following infusions of CAV-GFP, we observed cell bodies in numerous brain regions including the medial 
septum-nucleus of the diagonal band (MS-NDB) where the number of labeled cells appeared to be different in NG- mice after 5 months of reduced neurogenesis. MS-NDB was also particularly interesting since its hippocampal cholinergic projections are thought to regulate neurogenesis (Berg et al., 2013; Mohapel et al., 2005), be important for short-term memory (Chang and Gold, 2004), and exhibit deleterious changes during aging (Banuelos et al., 2013; Stroessner-Johnson et al., 1992). Compared to NG+ mice, NG- mice with a 5-month long reduction of neurogenesis had more GFP+ cells in the MS-NDB both ipsilateral and contralateral to the injection site (Figure 1B,C), indicating that additional MS-NDB neurons were projecting to the hilus in NGanimals. The change in the septohippocampal cholinergic inputs was not detectable in NGmice with a 2 month-long reduction in neurogenesis (Figure 1C), indicating that this type of remodeling requires extended periods of living without neurogenesis. The change in connectivity was not detected in another major hilus projection, the locus coeruleus (LC; Figure $1 \mathrm{~B}, \mathrm{C})$, indicating that reduction of neurogenesis causes changes in some, but not other hilar inputs. We wanted to verify that the circuit rewiring observed after 5 months with reduced neurogenesis was due to suppression of neurogenesis rather than an off-target effect of Xirradiation. We therefore used a genetic technique to suppress neurogenesis for 5 months in 2 month old mice and repeated our tracing experiments. New neurons generated in the subgranular zone develop from radial astrocytes that express glial fibrillary acidic protein (GFAP;(Garcia et al., 2004). We used mice that express herpes simplex virus thymidine kinase (Tk) under control of a GFAP promoter to suppress neurogenesis by administration of the antiviral drug valgancyclovir (VGCV) for 5 months. This approach was validated for targeting dividing stem cells and diminishing neurogenesis while sparing non-stem astrocytes (Garcia et al., 2004). After 5 months of VGCV treatment we observed suppression of neurogenesis in NG${ }^{\text {TK }}$ mice (GFAP-Tk+/- mice treated with VGCV for 5 months) compared to NG+ ${ }^{\text {TK }}$ (GFAP-Tk-/mice treated with VGCV for 5 months; Figure S1). We also observed that more cells bodies were labeled in the MS-NDB but not in the LC of NG- ${ }^{T K}$ mice compared to $\mathrm{NG}^{\mathrm{TK}}{ }^{\text {mice }}$ (Fig $1 \mathrm{C}$ ) 
echoing the results observed in X-irradiated mice. Together the results demonstrate rewiring of septohippocampal inputs in animals with diminished neurogenesis.

The MS-NDB hippocampal projection is comprised of roughly two thirds GABAergic, one third cholinergic and a small proportion of glutamatergic neurons (Amaral et al., 2007; Colom et al., 2005; Henderson et al., 2010). All three cell types project to the hilus (Amaral et al., 2007; Colom et al., 2005; Henderson et al., 2010). Remarkably, $80-90 \%$ of CAV-GFP labeled MSNDB cells in NG+, NG-, NG+ ${ }^{T K}$ and NG- ${ }^{T K}$ mice co-expressed the cholinergic marker choline acetyltransferase (ChAT), but not markers for the dominant GABAergic populations residing in the region (Figure $1 \mathrm{D}-\mathrm{E}$, Figure S2). Our results therefore signify that CAV primarily transduces cholinergic hilar inputs. In the autonomic nervous system mature neurons are occasionally thought to convert to a ChAT expressing phenotype (Apostolova et al., 2010). Moreover, recent reports suggest that CNS neurons can begin to express new identity markers in adult animals (Dulcis et al., 2013). We therefore also examined if the total number of ChAT+ cells was changed in animals living without neurogenesis. We found that the total number of cholinergic neurons in the MS-NDB was not different in NG+ and NG- mice or NG+ ${ }^{\mathrm{TK}}$ and NG${ }^{T K}$ mice (Figure S3), suggesting that the increase in labeled cells was due to recruitment of inputs into the region from already existing cholinergic neurons that project elsewhere in NG+ mice.

\section{Ongoing neurogenesis maintains working memory.}

Aging and chronic stress reduce neurogenesis (Zhao et al., 2008) while compromising cholinergic function (Nyakas et al., 2011; Terry and Buccafusco, 2003), and short-term memory (Zhao et al., 2008). Therefore, an increase in cholinergic inputs as a result of neurogenesis ablation seemed surprising. We therefore hypothesized that increased inputs in our models of reducing neurogenesis across aging reflected a natural compensation for an evolving 
impairment in cholinergic function. We tested NG- mice in a 4-arm spontaneous alternation task, which is dependent on an intact cholinergic septohippocampal projection(Chang and Gold, 2004). We found that NG+ and NG- mice performed at similar levels when neurogenesis was reduced for 2 and 4 months. However, a deficit in short-term memory emerged in NG- mice after 5 months, coinciding with changes in the septohippocampal projection (Chang and Gold, 2004). This deficit remained after 12 months without neurogenesis, when NG- animals performed at chance levels demonstrating a failure of working memory in this task (Figure 2 AE). Thus, a reduction of neurogenesis has a slowly emerging, but profound effect on a cholinergic-dependent working memory task .

Several learning and anxiety tasks have been examined in mice with short-term ablation of hippocampal neurogenesis(Drew et al., 2010). We confirmed reports that NG- mice have an impairment in a form of contextual fear conditioning after 8 weeks without neurogenesis, but display unimpaired anxiety-related behavior in the open field or elevated plus maze (Figure S4 and Figure $2 F, G$ ) neither of which depend on cholinergic functioning or neurogenesis.

Importantly, NG- animals were not impaired on a beam-walking task (Figure $2 \mathrm{H}$ ), which requires an intact cholinergic nucleus basalis projection to the frontoparietal cortex (Lehmann et al., 2002). This demonstrated that the reduction in neurogenesis alters the septohippocampal projection system, but not other cholinergic circuitry.

\section{Cholinergic dysfunction precedes input rewiring and memory deficits.}

Given the delayed emergence of our findings, we hypothesized that changes in septohippocampal circuit function precede our behavioral and anatomic observations. We tested this by challenging the cholinergic system in NG- mice after 2 months without neurogenesis with the muscarinic cholinergic antagonist scopolamine, which impairs spontaneous alternation behavior in high doses (Andriambeloson et al., 2014). As expected, at 
a normally sub-threshold dose, scopolamine did not affect NG+ mice, however it profoundly impaired spontaneous alternation behavior in NG- mice even after 2 months without neurogenesis (Figure 3A). This experiment indicates that cholinergic dysfunction is present in NG- animals long before behavioral changes emerge. It also supports the hypothesis that changes in septohippocampal inputs observed in Figure 1 may be compensatory to a primary loss in cholinergic tone. Since we observed no decrease in septal cholinergic neurons projecting to the hippocampus (Figure 1), we hypothesized that deficits in spontaneous alternation could be rescued by bolstering the endogenous cholinergic tone in NG- mice. We administered physostigmine, a cholinesterase inhibitor, which increases synaptic acetylcholine, to NG- mice and found that the spontaneous alternation deficit could be acutely and fully restored even after 12 months without neurogenesis (Figure 3B). Together these results strongly suggested a slowly emerging acetylcholine deficit in NG- mice.

We therefore used awake-behaving microdialysis and HPLC to directly measure hippocampal acetylcholine efflux both at baseline and during spontaneous alternation in mice with neurogenesis reduced for 4,5 and 7 months (Figure 3C). We observed that only a prolonged reduction in neurogenesis lead to reduced acetylcholine in the DG. Four months without neurogenesis resulted in no change in DG acetylcholine levels at baseline or during spontaneous alternation (Figure 3D). However, after 5 and 7 months without neurogenesis, NGmice exhibited reduced DG acetylcholine both at baseline and during spontaneous alternation (Figure 3E,F). Moreover, both NG+ and NG- groups at 4, 5 and 7 months mounted an increase in acetylcholine efflux while performing the spontaneous alternation task (Figure 3G-I), indicating that the cholinergic projection was engaged but the total release was attenuated. Interestingly, the 4 month group had a slightly attenuated acetylcholine increase while performing spontaneous alternation (Figure 3G). Together the results indicate that reduction in neurogenesis initially results in a subthreshold acetylcholine deficit, which is followed by 
attenuated release of phasic acetylcholine, progressing to a tonic efflux deficit and ultimately functional circuit deficits with compensatory changes in cholinergic innervation. Our findings therefore show that ongoing neurogenesis functions to maintain septohippocampal cholinergic tone and function.

\section{Ventral cholinergic projection is recruited by the dorsal hilus during rewiring.}

Normally, the septohippocampal projection has a highly organized topography. In rats, MS-NDB neurons project to either the dorsal or ventral hilus, but not to both (Ohara et al., 2013), though this has not been previously established in mice. We hypothesized that the increase in dorsally projecting MS-NDB cells in NG- mice after 5 months results from recruitment of cholinergic fibers of passage that normally traverse the dorsal to innervate the ventral DG. We analyzed the topography of MS-NDB cells projecting to the dorsal hilus in NG+ and NG- mice. We found that the increase in labeled cells in NG- mice after 5 months with reduced neurogenesis originated from structures that normally project to the ventral hippocampus, the medial NDB and lateral aspect of the MS in rats (Figure 4B). This suggested that, aging with reduced neurogenesis results in rewiring within the septohippocampal projection. We then directly tested our hypothesis by injecting a CAV-GFP into the dorsal hilus and a red reporter CAV (CAVCherry) into the ventral hilus in NG+ and NG- mice after 5 months of reduced neurogenesis (Figure 4C). We found that in NG+ mice, as in rats, dorsally projecting (GFP-labeled) cells were mostly distinct from ventrally projecting (Cherry labeled) cells (Figure 4D). However, in NGmice $\approx 40 \%$ of cells with terminals in the dorsal hilus also had terminals in the ventral hilus (GFP and Cherry labeled; Figure 4D). This result indicates a rewiring of the septohippocampal projection in NG- mice where ventrally projecting MS-NDB cholinergic neurons also develop dorsal projections. Moreover it appears that ventrally projecting MS-NDB cholinergic neurons also demonstrated rewiring within the ventral hippocampus. NG- mice showed an increased number of Cherry labeled cells in the MS-NDB, specifically in the ventrally projecting medial 
NDB and lateral MS compared to NG+ mice (Figure 4E). Therefore, aging without neurogenesis results in increased innervation, albeit dysfunctional, in both the ventral and the dorsal hippocampus by normally ventrally projecting MS-NDB neurons. The findings provide a mechanism for increased numbers of septal cholinergic inputs into the dorsal hippocampus of NG- mice.

\section{Discussion}

Our findings demonstrate a critical role for ongoing neurogenesis over extended periods of time in the maintenance of cholinergic hippocampal inputs and non-reinforced working memory. This represents a remarkably slow form of plasticity emerging over months. A temporal scale of such a magnitude corresponds to cumulative changes observed during chronic disease and aging. Accordingly, hippocampal neurogenesis is reliably reduced during chronic psychosocial stress, aging, and in Alzheimer's disease (Perry et al., 2012; Shors, 2008). Here, we have observed that an experimental chronic reduction in neurogenesis is sufficient to result in systems level hippocampal dysfunction over time.

The earliest evidence of subthreshold cholinergic deficits in our study corresponds to time points when numerous, more subtle behavioral deficits associated with ablation of neurogenesis have been described (Clelland et al., 2009; Drew et al., 2010; Snyder et al., 2011). Our findings therefore raise a possibility that mild cholinergic dysfunction may underlie the more subtle behavioral deficits more proximately following ablation of young neurons. Studies using physostygmine could be used to address this possibility.

Ultimately, a profound degradation of DG-dependent working memory emerged in NG- mice along with a dramatic remodeling of septohippocampal cholinergic DG inputs. Septal cholinergic neurons were previously demonstrated to undergo axonal sprouting in the absence of synaptic 
targets (Stanfield and Cowan, 1982) and temporary remodeling while animals are housed in enriched environments (Bergami et al., 2015). Our findings indicate that elimination of adultborn neurons results in compensatory rewiring of the septohippocampal projection by recruitment of ventrally projecting axons for dual (dorsal-ventral) innervation. Thus, young dentate granule neurons appear to maintain the integrity of the septohippocampal circuit over the lifespan. We hypothesize that hippocampal neurogenesis serves as a functional target for cholinergic septohippocampal neurons, and in their absence cholinergic cells initially show a reduction in cholinergic activity, then reorganize their innervation as a compensatory mechanism (Figure 4F).

The progressive nature of behavioral and anatomic changes presented here is reminiscent of neurodegenerative changes observed during age-related cognitive decline and in Alzheimer's disease, where decreased cholinergic innervation was previously linked to fewer neural stem cells (Terry and Buccafusco, 2003). Hence, neurogenesis may constitute a target for stabilizing the septohippocampal circuit and consequently working memory during normal aging and in disease states. More broadly, given the susceptibility of adult neurogenesis to the effects of chronic stress and aging, it is intriguing to speculate that the adult connectome is more malleable by experiences than currently appreciated. Our results raise the possibility that targeting distinct populations of cells constitutes a viable strategy for rewiring of long-range connectivity in the brain.

Author Contributions: Conceptualization, GSK and AD; Methodology GSK, LMS and AD; Validation, GSK. Formal analysis, GSK; Investigation, GSK, VKR, RMS and LMS; Resources, GSK; Data Curation, GSK; Writing - Original draft GSK and AD; Writing - Review and Editing, GSK, EDL and AD; Visualization, GSK, VKR and AD; Supervision EDL and AD; Project administration, GSK; Funding Acquisition, GSK, LMS, EDL and AD. 
Acknowledgements The authors thank Joshua Gordon and Steven Siegelbaum for critical reading of the manuscript and members of the ADL and Gordon labs for helpful insights. GSK was supported by a Canadian Institutes of Health Research Postdoctoral Fellowship. This work was supported by MH091844 and NARSAD young investigator award (AD), MH091427 (EDL), and NS085502 (LMS). AD is an Irving Scholar. Authors declare no conflicts of interest.

\section{Figure Legends:}

Figure 1. Increased cholinergic hilar inputs in mice living with diminished neurogenesis. (A) 2 month-old mice were exposed to focal hippocampal X-irradiation or VGCV. CAV-GFP was injected into the dorsal hilus 5 months after X-irradiation or VGCV treatment. CAV-GFP was injected also into a group of X-irradiated mice after two months. (B) GFP+ cells in the MS-NDB and the LC in NG+ and NG- mice ( $n=12$ per group). (C) NG- mice without neurogenesis for 5 months showed significantly more cells projecting to the dorsal hilus than NG+ mice from the MS-NDB both ipsilateral $(\mathrm{I})(\mathrm{t}(22)=2.238, \mathrm{p}=0.0357)$ and contralateral $(\mathrm{C} ; \mathrm{t}(22)=3.316, \mathrm{p}=$ $0.0033)$ to the injection site. NG- mice without neurogenesis for 2 months $(n=5)$ and NG+ mice $(n=5)$ showed similar connectivity from MS-NDB and LC to the dorsal hilus. NG- ${ }^{\text {TK }}$ mice with suppressed neurogenesis for 5 months showed significantly more cells projecting to the dorsal hilus than $\mathrm{NG}+{ }^{\mathrm{TK}}$ mice from the MS-NDB ipsilateral to the injection site $(\mathrm{t}(7)=2.927, \mathrm{p}=0.0221)$. $(D, E)$ In the MS-NDB $80-90 \%$ of GFP labeled cells overlap with a primary marker of cholinergic cells, acetylcholineserase in 5 and 2 month groups. I= Ipsilateral, C= Contralateral. Bars represent mean \pm SEM.

\section{Figure 2. A working memory deficit emerges in mice after a prolonged reduction of adult}

neurogenesis. (A) Behavior of NG- mice was assessed at 2, 4, 5 and 12 months without neurogenesis and compared to age matched NG+ controls. (B-E) Spontaneous alternation pattern (SAP) in a 4-arm spontaneous alternation task. (B,C) NG- mice after 2 and 4 months without neurogenesis had similar SAP scores of about $60 \%$ as NG+ mice ( 2 months $n=15$ per 
group, 4 months NG+ n=12 NG- n=13). (D) However, after 5 months without neurogenesis a deficit emerged in NG- mice where they showed SAP scores around $50 \%$ (NG+ n=10, NG$n=14 ; t(22)=3.835, p=0.0009)$. (E) After 12 months without neurogenesis SAP scores declined to chance levels of about $44 \%$ (dotted line) NG- mice (NG+ $n=7, N G-n=5 ; t(10)=4.06, p=$ 0.0023). (F) In the open field, NG- mice $(n=11)$ without neurogenesis for 5 months and NG+ mice $(n=8)$ showed no differences in total distance travelled in $30 \mathrm{~min}(\mathrm{~cm})$ or the percentage of time spent in the center. $(G)$ In the elevated plus maze NG- mice $(n=8)$ without neurogenesis for 5 months and NG+ mice $(n=11)$ showed similar open arm duration (s) and similar ratios of open to closed arm entries. $(H)$ In a beam walking task NG- mice $(n=6)$ without neurogenesis for 5 months and NG+ mice $(n=6)$ showed a similar number of total footslips and traversal latency (s). Bars represent mean \pm SEM.

Figure 3. Hippocampal acetylcholine release declines in mice after a prolonged reduction of adult neurogenesis. (A) NG- mice without neurogenesis for 2 months and NG+ mice were administered a muscarinic acetylcholine receptor antagonist scopolamine and spontaneous alternation pattern was assessed in a 4-arm spontaneous alternation task. Performance was compared across all groups (NG+C $n=15, N G-C n=15, N G+S n=5$ and NG- $S n=6$, main effect of group $\left.F_{3,37}=4.977, p=0.0053\right)$. The performance of NG- $S$ mice was reduced compared to all groups to near chance levels (dotted line). (B) NG- mice without neurogenesis for 12 months and NG+ mice were administered an acetlycholinesterase inhibitor physostigmine and spontaneous alternation pattern was assessed in a 4-arm spontaneous alternation task. Performance was compared across all groups (NG+C n=7, NG- C n=5, NG+P n=7, NG- P $n=5$, main effect of group $\left.F_{3,26}=8.683, p=0.0007\right)$. NG- $C$ mice performed below all groups and at chance. Bars represent mean \pm SEM, Tukey's post hoc ${ }^{*} p<0.05,{ }^{* *} p<0.01$. (C) Mice were treated with X-irradiation or Sham treatment and bilaterally implanted with cannulae. In one group of mice microdialysis measurements were taken from the left dorsal hilus after 4 months 
without neurogenesis. In a separate group of mice microdialysis measurements were taken from the right dorsal hilus at 5 months and the left dorsal hilus at 7 months without neurogenesis. Microdialysis measurements were taken at baseline (B1-B4), while mice were in the 4-arm spontaneous alternation maze (M1-M2) and after being removed from the maze (A1A2). (D) After 4 months without neurogenesis NG+ and NG- mice showed similar patterns of acetylcholine release that increased when animals were in the maze (NG+ n=7,NG- $n=8$; main effect of time $\left.F_{7,91}=8.504, p<0.0001\right)$. (E) After 5 months without neurogenesis NG- mice showed significantly lower acetylcholine release ( $n=6$ per group; main effect of group $F_{1,10}=$ 5.109, $p=0.0473$ ), main effect of time $F_{5,50}=10.87, p<0.0001$, interaction effect $F_{5,50}=4.198$, $p=0.0029,(F)$ Reduced acetylcholine in NG- mice remained after 7 months with reduced neurogenesis $\left(N G+n=5\right.$, NG- $n=6$; main effect of group $F_{1,9}=7.31, p=0.0242$, main effect of time $F_{7,63}=13.44, p<0.0001$, interaction effect $\left.F_{7,63}=6.904, p<0.0001\right)$. (G) Mice without neurogenesis for 4 months demonstrated an increase in acetylcholine above baseline during the spontaneous alternation task. NG- mice showed a slightly attenuated increase in acetylcholine while performing spontaneous alternation compared to $\mathrm{NG}+$ controls (main effect of time $\mathrm{F}_{7,91}=$ 8.921, $p<0.0001$, main effect of group $F_{7,91}=1.874, p=0.1942$, time $x$ group interaction $F_{7,91}=$ 1.786, $p=0.0996)(H)$ Mice without neurogenesis for 5 months demonstrated an increase in acetylcholine above baseline during the spontaneous alternation task ( main effect of time $F_{5,40}=$ 3.695, $p=0.0076$ ) as did animals after 7 months without neurogenesis (main effect of time $F_{7,63}$ $=10,68, p<0.0001)(I)$. Bars and points represent mean \pm SEM, Bonferroni post hoc * $p<0.05,{ }^{*}$ $p<0.01$

\section{Figure 4. Rewiring of cholinergic septotemporal projection after a prolonged reduction in} adult neurogenesis. (A) Mice were treated with X-irradiation or Sham treatment and CAV-GFP was injected into the dorsal hilus after 5 or 2 months. (B) After 5 months without neurogenesis, the dorsal hilus of NG- mice shows an increase in innervation by GFP+ cell bodies that are 
expected to primarily innervate the ventral hilus (Ohara et al., 2013) compared to NG+ mice ( $n=12$ per group). NG+ mice show an increase in projections to the dorsal hilus from the medial NDB, contralateral $(t(22)=3.171, p=0.0044)$ and ipsilateral $(t(22)=3.346, p=0.0029)$ to the injection site, as well as the lateral MS $(t(22)=2.554, p=0.0181)$. This change has not emerged in NG- mice without neurogenesis for 2 months. (C) In NG+ and NG- mice without neurogenesis for 5 months, CAV-GFP was injected into the dorsal hilus and CAV-Cherry was injected into the ventral hilus. $(E)$ In NG+ mice $(n=5) 10 \%$ of GFP+ cells in the MS-NDB showed Cherry labeling and this significantly increased to $40 \%$ in NG- mice $(n=4 ; t(7)=5.985, p=$ 0.0006). (E) NG+ mice $(n=5)$ show a greater number of cells projecting from the MS-NDB to the ventral hilus compared to NG- mice $(n=4, t(7)=4.754, p=0.0021)$. The increase in cell bodies is in the medial NDB $(t(7)=3.331, p=0.0126)$ and lateral $M S(t(7)=11.14, p<0.0001)$. Bars represent mean \pm SEM. ${ }^{*} p<0.05,{ }^{* *} p<0.01(F)$ Structural and functional reorganization of the septohippocampal circuit in NG- mice. NG+ mice show acetylcholine release that supports working memory and cholinergic afferent organization within the septohippocampal projection. NG- mice without neurogenesis for 2 months show an emerging deficit in acetylcholine release in the hippocampus but maintain cholinergic afferent organization within the septohippocampal projection. NG- mice without neurogenesis for 5 months show significant reductions in hippocampal acetylcholine release and rewiring of cholinergic septohippocampal inputs with septal neurons that normally project to the ventral hilus innervating the dorsal hilus and increased innervation of the ventral hilus. 


\section{Experimental Procedures}

\section{Animals}

Focal X-ray treatment of the hippocampus leads to long-term reductions of cell proliferation in the dentate gyrus and spares irradiation to most of the brain(Santarelli et al., 2003). Male C57BL/6J mice (Jackson Laboratories) were used in experiments of NG+ (sham) and NG- (Xirradiated) mice. Mice were delivered to our animal facility at 7-weeks-old and acclimated for one week before sham or X-irradiation treatment at 8 weeks.

GFAP-Tk heterozygous mice (NG- ${ }^{\text {TK}}$; (Bush et al., 1998) express herpes thymidine kinase (Tk) under control of the glial fibrillary acidic protein (GFAP) promoter, expressed in stem and nonstem astrocytes. Treatment with Valgancyclovir (VGCV) in NG- ${ }^{T K}$ mice leads to reductions in cell proliferation in dividing stem cells with relative sparing of non-stem astrocytes (Garcia, 2004). Female GFAP-Tk heterozygous mice were mated with wild-type littermate males, all on C57BL/6J $129 S 6$ mixed background. Male pups were genotyped using previously reported PCR reactions (Bush et al., 1998), weaned at P21, and housed 3-5 per cage with mixed genotypes. Half the mice were GFAP-Tk heterozygous $\left(\mathrm{NG}^{\mathrm{TK}}\right)$ and half were negative for the gene $\left(\mathrm{NG}+{ }^{\mathrm{TK}}\right.$ ). At 8 weeks mice were started on a feeding schedule of chow containing VGCV.

Mice were given ad libitum access to food and water under a 12:12 h light:dark cycle in a temperature-controlled $\left(72^{\circ} \mathrm{F}\right)$ colony. All animal experiments were performed in accordance with the Guide for the Care and Use of Laboratory Animals and approved by the New York State Psychiatric Institute Animal Care and Use Committee.

\section{X-irradiation}

Similar to previous studies(Santarelli et al., 2003), 8-week-old mice were anesthetized with ketamine and xylazine (150 mg/kg and $10 \mathrm{mg} / \mathrm{kg}$ respectively). NG+ mice were untreated. NG- 
mice were placed in a stereotaxic frame, covered by a lead shield with a $3.22 \times 11 \mathrm{~mm}$ opening over the hippocampus (interaural 3.00 to 0.00 ) and placed in a X-RAD 320 biological irradiator (PXI; North Branford, CT). The X-RAD 302 operated at $300 \mathrm{kV}$ and $12 \mathrm{~mA}$ with a 2-mm Al filter and delivered 2.5-Gy doses per X-ray session. Mice were treated for three sessions, separated by a 2 day interval (day 1, 4 and 7) so NG- mice received a total dose of 7.5-Gy. To assess the effect of X-ray on cell proliferation, tissue from NG+ and NG- mice were immunolabeled for a marker for cell division $\mathrm{Ki}-67$ and a marker of immature neurons DCX.

\section{Valgancyclovir treatment in GFAP-Tk ${ }^{+/-}$Mice}

Chow containing VGCV $(165 \mathrm{mg} / \mathrm{kg})$ was administered to mice at 8 week until 5 months of age. Mice were on a feeding schedule of chow where they were fed VGCV chow for 5 days and normal chow for 2 days. This feeding schedule was employed to reduce gastrointestinal side effects caused by Tk expression in gut tissues. To assess the effect of VGCV on cell proliferation, tissue from GFAP-Tk ${ }^{-/-}\left(\mathrm{NG}^{\mathrm{TK}}\right)$ and GFAP-Tk ${ }^{+/-}\left(\mathrm{NG}^{-{ }^{\mathrm{TK}}}\right)$ mice were immunolabeled for a marker for cell division Ki-67 and a marker of immature neurons DCX.

\section{Retrograde Tracing}

To visualize cell bodies projecting to the dorsal dentate gyrus, we used a canine adenovirus, expressing GFP (CAV-GFP), which is taken up by axon terminals and transported to cell bodies(Kissa et al., 2002). NG+ and NG- mice at 5 or 2 months after treatment were used. Mice were anesthetized with a ketamine, xylazine, acepromazine mixture $(65 \mathrm{mg} / \mathrm{kg}, 13 \mathrm{mg} / \mathrm{kg}$, $1.5 \mathrm{mg} / \mathrm{kg}$ respectively) and placed into a stereotaxic frame (David Koph Instruments) with the skull exposed. A $10 \mu \mathrm{l} \mathrm{Hamilton}$ syringe with pulled glass pipette was used to infuse $1.5 \mu$ of CAV-GFP $\left(5 \times 10^{12}\right)$ to the right dorsal hilus (bregma coordinates: anteroposterior $-2.3 \mathrm{~mm}$, 
mediolateral $1.6 \mathrm{~mm}$, dorsoventeral $-1.6 \mathrm{~mm}$ ) at $0.2 \mu \mathrm{l} / \mathrm{min}$. Mice were sacrificed $1-4$ weeks following surgery.

To visualize cell bodies projecting to the dorsal hippocampus and the ventral hippocampus, we infused CAV-GFP in the dorsal hippocampus and CAV-cherry in the ventral hippocampus. A group of 7-month-old NG+ and NG- mice were infused with CAV-GFP in the right dorsal hilus as described above. In addition the right ventral hilus (bregma coordinates: anteroposterior $3.2 \mathrm{~mm}$, mediolateral $2.3 \mathrm{~mm}$, dorsoventeral $-4.3 \mathrm{~mm})$ was infused with $1.5 \mu \mathrm{l}$ of CAV-cherry $(5 \mathrm{x}$ $10^{12}$ ) at $0.2 \mu \mathrm{l} / \mathrm{min}$. Mice were sacrificed $1-4$ weeks following surgery.

\section{Immunohistochemistry}

Mice were anesthetized with a ketamine and xylazine mixture $(150 \mathrm{mg} / \mathrm{kg}$ and $10 \mathrm{mg} / \mathrm{kg}$ respectively) and transcardially perfused with ice cold phosphate-buffered saline (PBS; $\mathrm{pH} 7.4$ ) followed by $4 \%$ paraformaldehyde (PFA) in PBS. Brains were stored in 4\% PFA overnight and transferred to $30 \%$ sucrose for $48 \mathrm{~h}$. Brains were sagittally sectioned at $35 \mu \mathrm{m}$ and stored in PBS with $0.02 \%$ azide.

For immunostaining tissue was washed in PBS, blocked with 10\% normal donkey serum and incubated in primary antibody overnight at $4^{\circ} \mathrm{C}$. The following primary antibodies were used and diluted in 10\% normal donkey serum: rabbit Ki67 (1:100 Vector laboratories), goat DCX (1:500; Santa Cruz Biotechnology), chicken GFP (1:500; Abcam), rabbit Living Colors $®$ DsRed Polyclonal Antibody (1:1000; Clontech). Neurotrace (Life Technologies) served as a counterstain. All fluorescent secondary antibodies were obtained from Jackson ImmunoResearch and diluted 1:200 in PBS.

As outlined in the Allen Reference atlas(Lein et al., 2007), 20 sagittal sections (S1-S20) of each hemisphere per mouse spaced at $200 \mu \mathrm{m}$ intervals was analyzed. GFP or Cherry labeled cells 
in the lateral NDB were counted in sections S13-S15. GFP or Cherry cells in the medial NDB were counted in sections S16-S18. GFP or Cherry cells in the lateral MS were counted in section S20. We did not collect consistent intact samples of section S21 and could not assess the medial MS. For the LC, GFP cells were counted in section S15. LC cells were only detected ipsilateral to the injection. Cherry cells were only detected in the ipsilateral hemisphere. To normalize for viral infection efficiency in each individual mouse, the number of cells in the region of interest (MS, NDB or LC) was divided by the number of cells adjacent to the viral injection site in the dentate gyrus. GFP or Cherry cells were counted in the dentate gyrus, in sections S7 and S8 and averaged. All GFP or Cherry cells in the MS-NDB in sections S13-S20 were assessed for ChAT co-expression and total ChAT cells. DCX and Ki67 were counted unilaterally in 5 sections of dentate gyrus that spanned the septotemporal axis

\section{Imaging and analysis}

Tissue was imaged at 20x on a fluorescent microscope (Olympus IX83). The Allen Brain Atlas was used to define brain regions in sagittal sections. GFP or cherry expressing cells were counted in the medial septum and the diagonal band ipsilateral and contralateral to the injection in sections that transversed the structure.

\section{Behavioral experiments}

All behavioral experiments were performed by a female scientist and took place during the light cycle between 9AM and 3PM. Mice with reduced neurogenesis for 2 months were tested in the following order: spontaneous alternation, open field, fear conditioning. Mice with reduced neurogenesis for 5 months were tested in the following order: spontaneous alternation, open field, elevated plus maze, balance beam. Mice without neurogenesis for 4 months were tested 
for spontaneous alternation. A group of mice without neurogenesis for 2 months and 12 months were tested in spontaneous alternation and used for pharmacological experiments.

\section{Spontaneous alternation}

To assess spontaneous alternation mice were tested in a closed arm plus maze as described with modifications(Chang and Gold, 2004). The plus maze consisted of four identical arms (25 $\times 5 \times 30 \mathrm{~cm}$ ) with opaque walls that extended from a center platform $(5 \times 5 \mathrm{~cm})$ elevated $50 \mathrm{~cm}$ from the floor. Testing occurred in a lit (250 lux) room. Mice were placed on the center platform and allowed to explore freely for $12 \mathrm{~min}$. The sequence of arm entries was scored throughout the 12 min. A successful alternation occurred when a mouse made four discrete arm entries on overlapping sets of five consecutive entries. Accordingly the number of successful alternations is the total number of entries minus four. The spontaneous alternation score is calculated by (successful alternations/total possible alternations)x 100 ; a score of $44 \%$ reflects chance performance.

\section{Open Field}

Mice were placed in a Plexiglas open field (Kinder Scientific SmartFrame 22.1" x 22.1" x 15.83") illuminated by 80-100 lux for thirty minutes. Behavioral measures were automatically recorded by infrared photo beams and analyzed by MotorMonitor software.

\section{Elevated-plus maze}

The elevated plus maze was performed as described(Avgustinovich et al., 2000), and consisted of a central platform $(5 \times 5 \mathrm{~cm})$ with two opposing open arms $(25 \times 5 \mathrm{~cm})$ and two opposing arms enclosed by opaque walls $(25 \times 5 \times 30 \mathrm{~cm})$, elevated $50 \mathrm{~cm}$ from the floor. Experiments were conducted in the dark with open arms illuminated (100-120 lux). Mice were placed on the 
central platform facing a closed arm; the number of entries to each arm and duration in each arm was scored for 5 min by an experienced observer.

\section{Balance Beam}

Beam walking was assessed as described(Xie et al., 2010). Mice were given 5 training trials where they traversed a $100 \mathrm{~cm}$ long, $1.5 \mathrm{~cm}$ diameter circular beam in a lit room (250 lux). 24 hours after training mice traversed the beam once while the number of foot slips and latency to cross the beam were scored.

\section{Microdialysis}

We performed microdialysis to measure baseline and spontaneous alternation induced acetylcholine levels in the dorsal hippocampus as previously described with modifications(Chang and Gold, 2004). NG+ and NG- mice aged without neurogenesis for 1.5 months or 4.5 months were implanted bilaterally with microdialysis guide cannulae (Synaptech, S-3000) in the dorsal hippocampus (bregma coordinates: anteroposterior $-2.3 \mathrm{~mm}$, mediolateral $1.6 \mathrm{~mm}$, dorsoventeral $-0.6 \mathrm{~mm}$ ). The cannulae were implanted $1 \mathrm{~mm}$ above the dorsal hilus target region as we used a $1 \mathrm{~mm}$ membrane to sample from the dorsal hilus. The cannulae were secured to the skull with skull screws and dental cement and mice recovered from surgery for at least 2 weeks.

Microdialysis samples were collected at baseline, during spontaneous alternation and after spontaneous alternation. One group of NG+ and NG- mice were tested after 4 months without neurogenesis with a probe in the left hippocampus. Another group of NG+ and NG- mice were tested after 5 months without neurogenesis with a probe in the right hippocampus and again after 7 months without neurogenesis with a probe in the left hippocampus. 
To begin a trial, a microdialysis probe with a $1 \mathrm{~mm}$ membrane (Synaptech, S3010 Synaptech Technology Inc., Marquette, MI) was inserted into the dorsal hilus at the same coordinates as the dorsally infused CAV-GFP (bregma coordinates: anteroposterior $-2.3 \mathrm{~mm}$, mediolateral $1.6 \mathrm{~mm}$, dorsoventeral $-1.6 \mathrm{~mm}$ ) and mice were placed in an opaque holding cage with fresh bedding. The probes were continuously perfused with $100 \mathrm{nM}$ neostigmine bromide (Sigma) in artificial cerebrospinal fluid (aCSF; $128 \mathrm{mM} \mathrm{NaCl}, 2.5 \mathrm{mM} \mathrm{KCl}, 1.3 \mathrm{mM} \mathrm{CaCl}_{2}, 2.1 \mathrm{mM} \mathrm{MgCl}$, $0.9 \mathrm{mM} \mathrm{NaH}_{2} \mathrm{PO}_{4}, 2.0 \mathrm{mM} \mathrm{NaHPO}_{4}$, and $1.0 \mathrm{mM}$ glucose at a $\mathrm{pH}$ of 7.4 ) at $1 \mu \mathrm{l} / \mathrm{min}$. For the first 60 min mice acclimated to the probe and dialysate was not collected. After this period dialysate samples were collected every $6 \mathrm{~min}$. The first 4 baseline samples were collected while the mouse was in the holding cage. After the baseline sampling time mice were placed in the spontaneous alternation task for 12 min while 2 samples were collected. Finally mice were placed back into the holding cage while 2 post-maze samples were collected.

\section{HPLC}

Dialysate samples were assayed for acetylcholine using HPLC with electrochemical detection (Eicom USA, San Diego, CA). Acetylcholine peaks were quantified by comparison to peak heights of standard solutions and corrected for in vitro recovery of the probe. The system detection limit is reliably 5 femtomole of acetylcholine. Chromatographs obtained every 15 min/sample were analyzed using the software program Envision (provided by Eicom, USA).

\section{Pharmacology}

Scopolamine hydrobromide (Sigma), $0.005 \mathrm{mg} / \mathrm{kg}$ dissolved in $0.9 \%$ saline was delivered i.p. 40 min prior to the spontaneous alternation task.

Physostigmine hemisulfate (Tocris) $20 \mathrm{ug} / \mathrm{kg}$ dissolved in $0.9 \%$ saline was delivered i.p. $15 \mathrm{~min}$ prior to the spontaneous alternation task. 


\section{Statistics}

All statistics were calculated in GraphPad Prism. All data are presented as the means \pm SEM and significance was set at $p<0.05$. ANOVAs that yielded statistically significant main effects were followed with Bonferroni or Tukey's post hoc tests. 


\section{References}

Amaral, D.G., Scharfman, H.E., and Lavenex, P. (2007). The dentate gyrus: fundamental neuroanatomical organization (dentate gyrus for dummies). Progress in brain research 163, 322.

Andriambeloson, E., Huyard, B., Poiraud, E., and Wagner, S. (2014). Methyllycaconitine- and scopolamine-induced cognitive dysfunction: differential reversal effect by cognition-enhancing drugs. Pharmacol Res Perspect 2, e00048.

Apostolova, G., Loy, B., Dorn, R., and Dechant, G. (2010). The sympathetic neurotransmitter switch depends on the nuclear matrix protein Satb2. The Journal of neuroscience : the official journal of the Society for Neuroscience 30, 16356-16364.

Avgustinovich, D.F., Lipina, T.V., Bondar, N.P., Alekseyenko, O.V., and Kudryavtseva, N.N. (2000). Features of the genetically defined anxiety in mice. Behav Genet 30, 101-109.

Banuelos, C., LaSarge, C.L., McQuail, J.A., Hartman, J.J., Gilbert, R.J., Ormerod, B.K., and Bizon, J.L. (2013). Age-related changes in rostral basal forebrain cholinergic and GABAergic projection neurons: relationship with spatial impairment. Neurobiol Aging 34, 845-862.

Berg, D.A., Belnoue, L., Song, H., and Simon, A. (2013). Neurotransmitter-mediated control of neurogenesis in the adult vertebrate brain. Development 140, 2548-2561.

Bergami, M., Masserdotti, G., Temprana, S.G., Motori, E., Eriksson, T.M., Gobel, J., Yang, S.M., Conzelmann, K.K., Schinder, A.F., Gotz, M., and Berninger, B. (2015). A critical period for experience-dependent remodeling of adult-born neuron connectivity. Neuron 85, 710-717. Bush, T.G., Savidge, T.C., Freeman, T.C., Cox, H.J., Campbell, E.A., Mucke, L., Johnson, M.H., and Sofroniew, M.V. (1998). Fulminant jejuno-ileitis following ablation of enteric glia in adult transgenic mice. Cell 93, 189-201. 
Chang, Q., and Gold, P.E. (2004). Impaired and spared cholinergic functions in the hippocampus after lesions of the medial septum/vertical limb of the diagonal band with 192 lgGsaporin. Hippocampus 14, 170-179.

Clelland, C.D., Choi, M., Romberg, C., Clemenson, G.D., Jr., Fragniere, A., Tyers, P., Jessberger, S., Saksida, L.M., Barker, R.A., Gage, F.H., and Bussey, T.J. (2009). A functional role for adult hippocampal neurogenesis in spatial pattern separation. Science 325, 210-213. Colom, L.V., Castaneda, M.T., Reyna, T., Hernandez, S., and Garrido-Sanabria, E. (2005). Characterization of medial septal glutamatergic neurons and their projection to the hippocampus. Synapse 58, 151-164.

Drew, M.R., Denny, C.A., and Hen, R. (2010). Arrest of adult hippocampal neurogenesis in mice impairs single- but not multiple-trial contextual fear conditioning. Behav Neurosci 124, 446-454.

Dulcis, D., Jamshidi, P., Leutgeb, S., and Spitzer, N.C. (2013). Neurotransmitter switching in the adult brain regulates behavior. Science 340, 449-453.

Garcia, A.D., Doan, N.B., Imura, T., Bush, T.G., and Sofroniew, M.V. (2004). GFAP-expressing progenitors are the principal source of constitutive neurogenesis in adult mouse forebrain. Nature neuroscience 7, 1233-1241.

Henderson, Z., Lu, C.B., Janzso, G., Matto, N., McKinley, C.E., Yanagawa, Y., and Halasy, K. (2010). Distribution and role of Kv3.1b in neurons in the medial septum diagonal band complex. Neuroscience 166, 952-969.

Kissa, K., Mordelet, E., Soudais, C., Kremer, E.J., Demeneix, B.A., Brulet, P., and Coen, L. (2002). In vivo neuronal tracing with GFP-TTC gene delivery. Mol Cell Neurosci 20, 627-637. Knoth, R., Singec, I., Ditter, M., Pantazis, G., Capetian, P., Meyer, R.P., Horvat, V., Volk, B., and Kempermann, G. (2010). Murine features of neurogenesis in the human hippocampus across the lifespan from 0 to 100 years. PloS one 5, e8809. 
Kuhn, H.G., Dickinson-Anson, H., and Gage, F.H. (1996). Neurogenesis in the dentate gyrus of the adult rat: age-related decrease of neuronal progenitor proliferation. The Journal of neuroscience : the official journal of the Society for Neuroscience 16, 2027-2033.

Lehmann, O., Jeltsch, H., Lazarus, C., Tritschler, L., Bertrand, F., and Cassel, J.C. (2002).

Combined 192 IgG-saporin and 5,7-dihydroxytryptamine lesions in the male rat brain: a neurochemical and behavioral study. Pharmacology, biochemistry, and behavior 72, 899-912. Lein, E.S., Hawrylycz, M.J., Ao, N., Ayres, M., Bensinger, A., Bernard, A., Boe, A.F., Boguski, M.S., Brockway, K.S., Byrnes, E.J., et al. (2007). Genome-wide atlas of gene expression in the adult mouse brain. Nature 445, 168-176.

Mohapel, P., Leanza, G., Kokaia, M., and Lindvall, O. (2005). Forebrain acetylcholine regulates adult hippocampal neurogenesis and learning. Neurobiol Aging 26, 939-946.

Nyakas, C., Granic, I., Halmy, L.G., Banerjee, P., and Luiten, P.G. (2011). The basal forebrain cholinergic system in aging and dementia. Rescuing cholinergic neurons from neurotoxic amyloid-beta42 with memantine. Behav Brain Res 221, 594-603.

Ohara, S., Sato, S., Tsutsui, K., Witter, M.P., and lijima, T. (2013). Organization of multisynaptic inputs to the dorsal and ventral dentate gyrus: retrograde trans-synaptic tracing with rabies virus vector in the rat. PloS one 8 , e78928.

Perry, E.K., Johnson, M., Ekonomou, A., Perry, R.H., Ballard, C., and Attems, J. (2012). Neurogenic abnormalities in Alzheimer's disease differ between stages of neurogenesis and are partly related to cholinergic pathology. Neurobiol Dis 47, 155-162.

Santarelli, L., Saxe, M., Gross, C., Surget, A., Battaglia, F., Dulawa, S., Weisstaub, N., Lee, J., Duman, R., Arancio, O., et al. (2003). Requirement of hippocampal neurogenesis for the behavioral effects of antidepressants. Science 301, 805-809.

Shors, T.J. (2008). From stem cells to grandmother cells: how neurogenesis relates to learning and memory. Cell Stem Cell 3, 253-258. 
Snyder, J.S., Soumier, A., Brewer, M., Pickel, J., and Cameron, H.A. (2011). Adult hippocampal neurogenesis buffers stress responses and depressive behaviour. Nature 476, 458-461.

Spalding, K.L., Bergmann, O., Alkass, K., Bernard, S., Salehpour, M., Huttner, H.B., Bostrom, E., Westerlund, I., Vial, C., Buchholz, B.A., et al. (2013). Dynamics of hippocampal neurogenesis in adult humans. Cell 153, 1219-1227.

Stanfield, B.B., and Cowan, W.M. (1982). The sprouting of septal afferents to the dentate gyrus after lesions of the entorhinal cortex in adult rats. Brain Res 232, 162-170.

Stroessner-Johnson, H.M., Rapp, P.R., and Amaral, D.G. (1992). Cholinergic cell loss and hypertrophy in the medial septal nucleus of the behaviorally characterized aged rhesus monkey. The Journal of neuroscience : the official journal of the Society for Neuroscience 12, 1936-1944. Terry, A.V., Jr., and Buccafusco, J.J. (2003). The cholinergic hypothesis of age and Alzheimer's disease-related cognitive deficits: recent challenges and their implications for novel drug development. J Pharmacol Exp Ther 306, 821-827.

van Praag, H., Shubert, T., Zhao, C., and Gage, F.H. (2005). Exercise enhances learning and hippocampal neurogenesis in aged mice. The Journal of neuroscience : the official journal of the Society for Neuroscience $25,8680-8685$.

Xie, G., Harrison, J., Clapcote, S.J., Huang, Y., Zhang, J.Y., Wang, L.Y., and Roder, J.C. (2010). A new Kv1.2 channelopathy underlying cerebellar ataxia. J Biol Chem 285, 3216032173.

Zhao, C., Deng, W., and Gage, F.H. (2008). Mechanisms and functional implications of adult neurogenesis. Cell 132, 645-660. 
bioRxiv preprint doi: https://doi.org/10.1101/311423; this version posted April 30, 2018. The copyright holder for this preprint (which was not certified by peer review) is the author/funder, who has granted bioRxiv a license to display the preprint in perpetuity. It is made available under aCC-BY-NC-ND 4.0 International license.
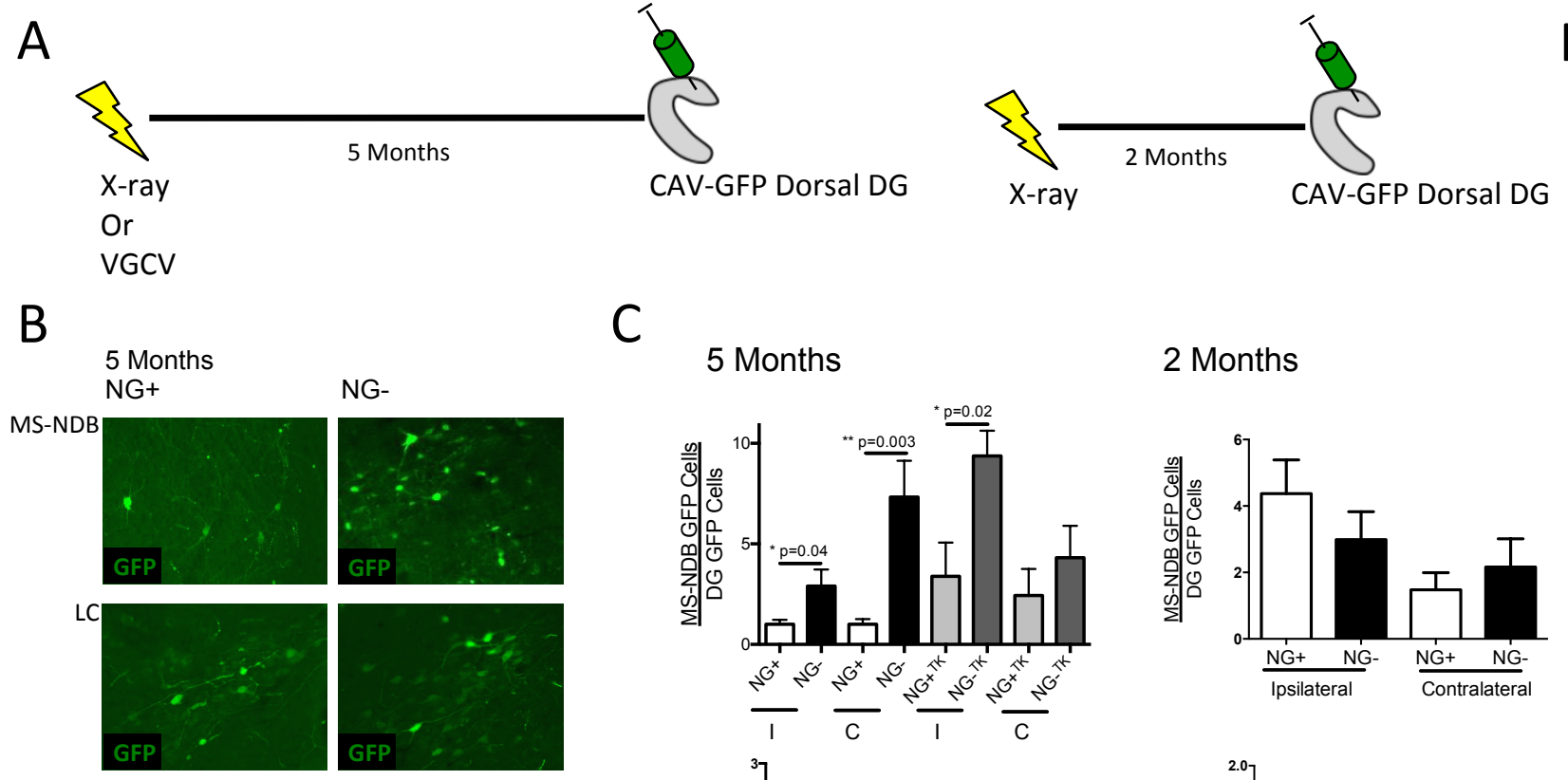

Figure 1

C

$$
5 \text { Months } 2 \text { Months }
$$
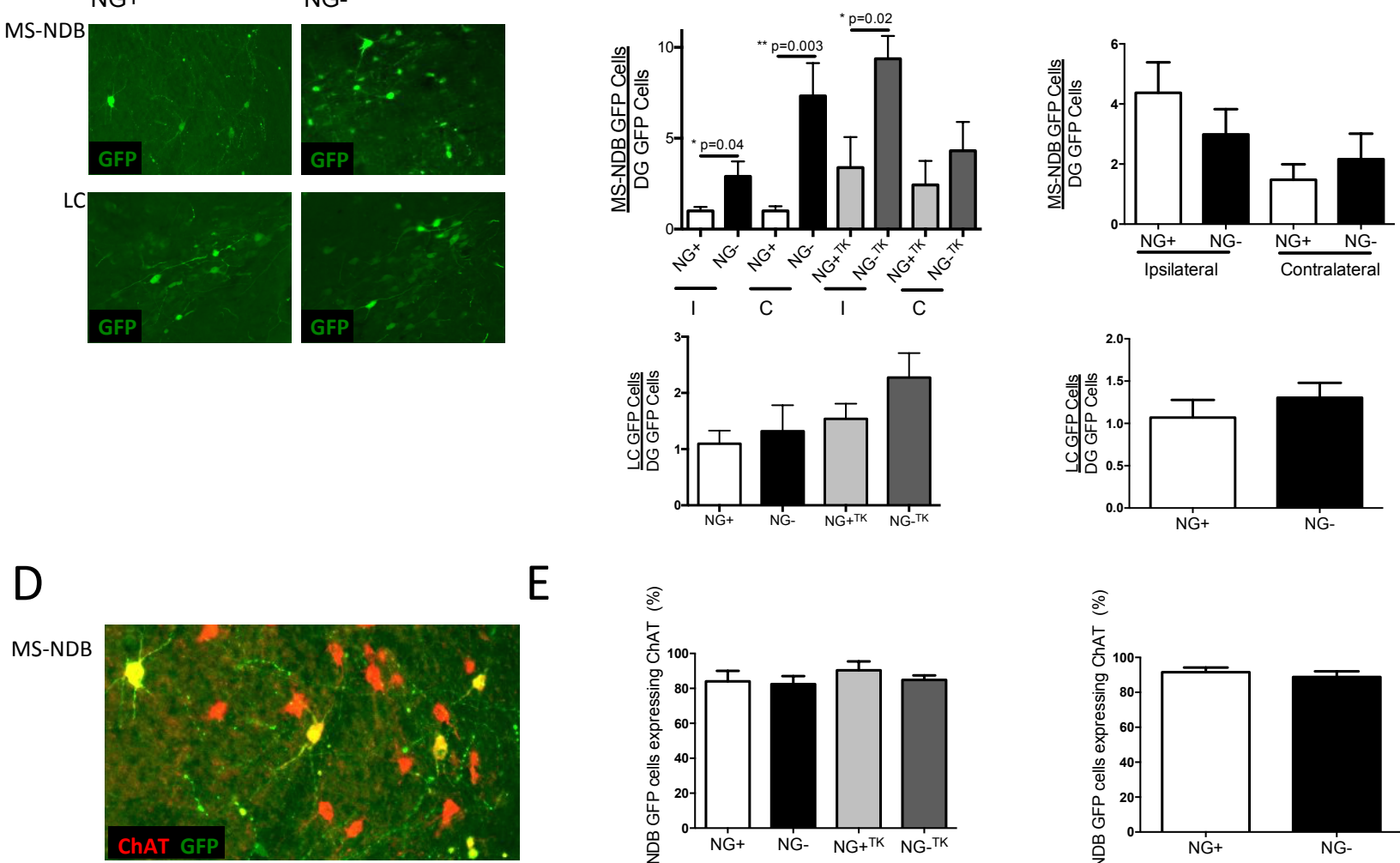

$\mathrm{E}$
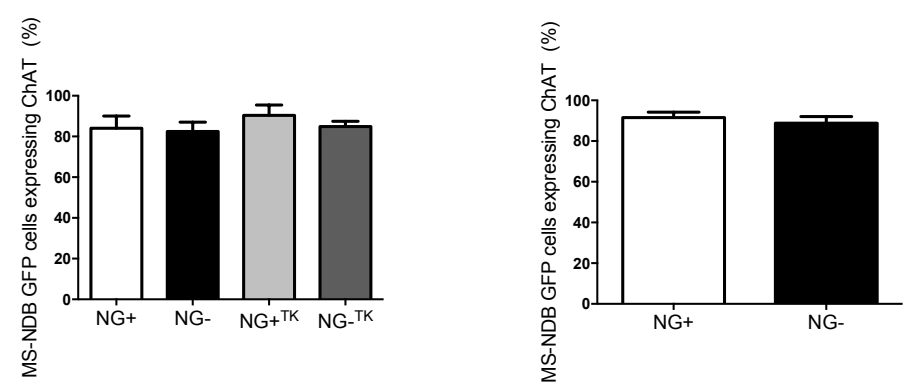
bioRxiv preprint doi: https://doi.org/10.1101/311423; this version posted April 30, 2018. The copyright holder for this preprint (which was not certified by peer review) is the author/funder, who has granted bioRxiv a license to display the preprint in perpetuity. It is made available under aCC-BY-NC-ND 4.0 International license.

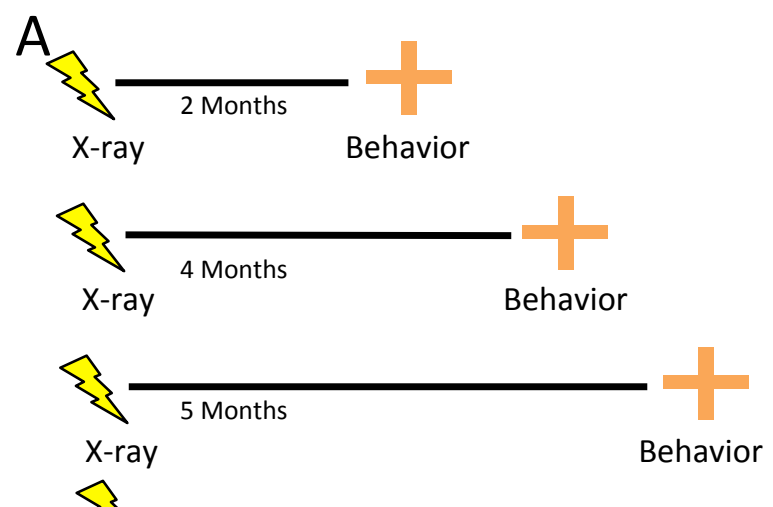

12 Months

X-ray

B

Spontaneous Alternation

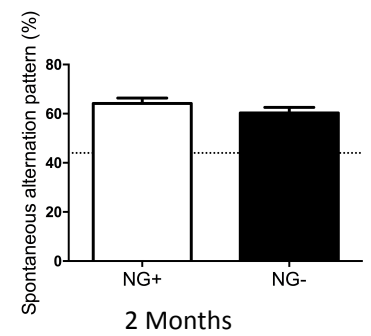

$\mathrm{F}$

Open Field 5 Months

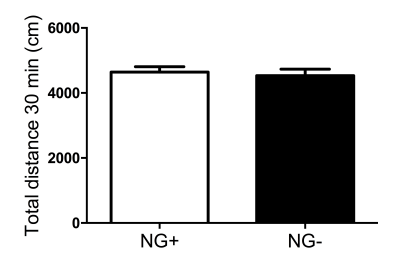

$\mathrm{H}$

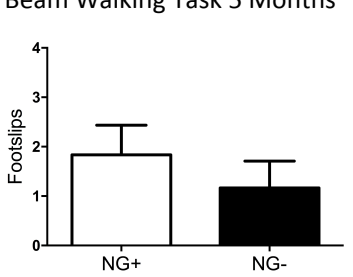

C

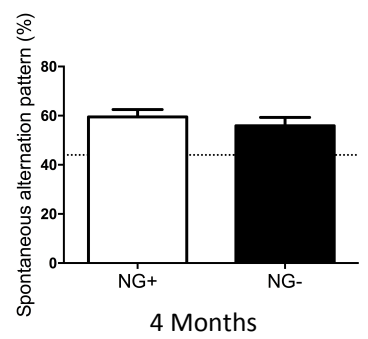

G
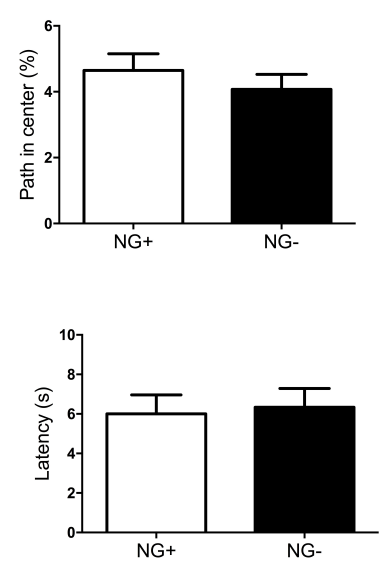

Figure 2

E
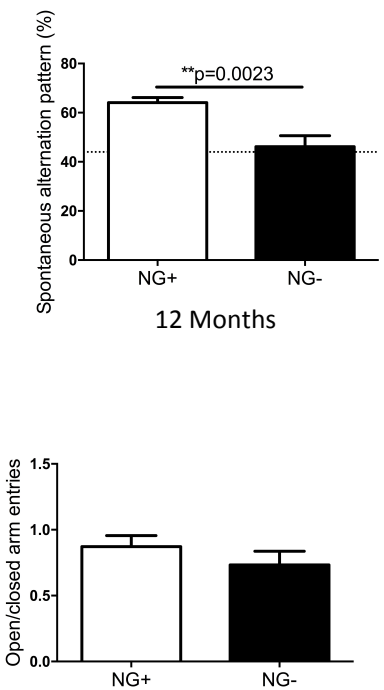

Elevated Plus Maze 5 Months

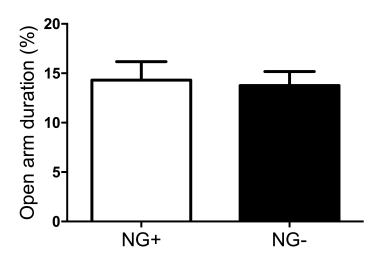

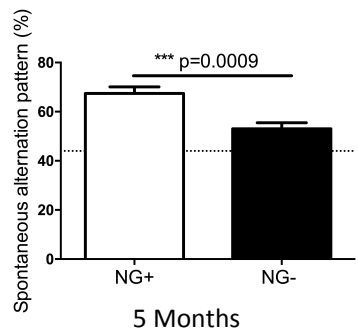

12 Months 
A

C

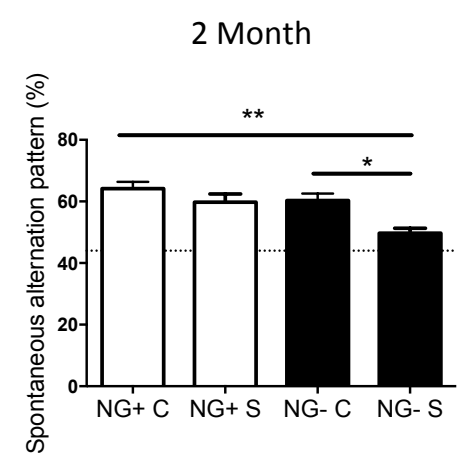

B

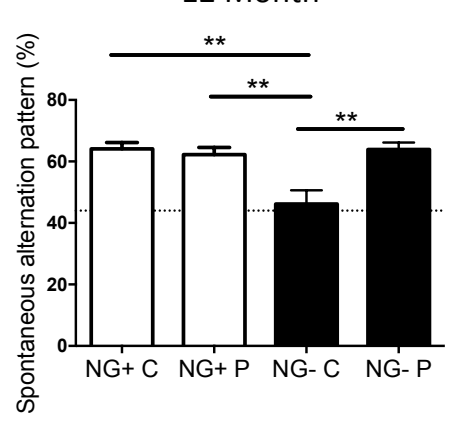

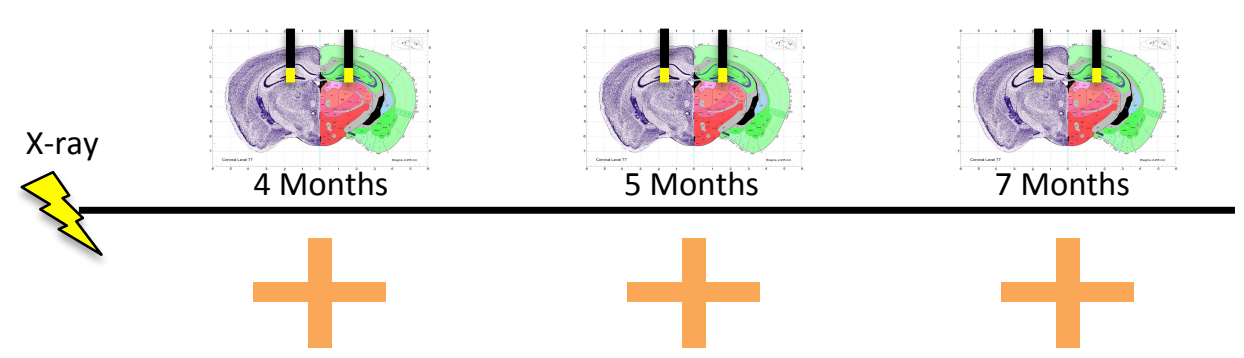

D

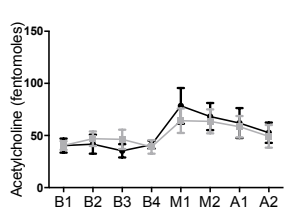

E
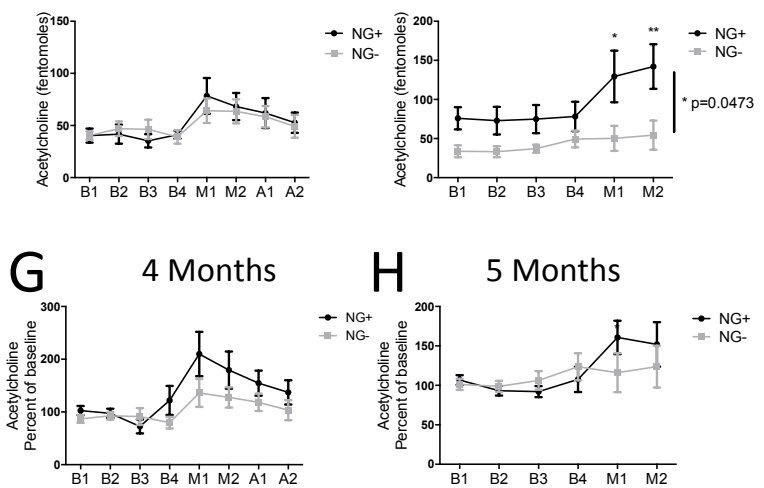

$\mathrm{F}$

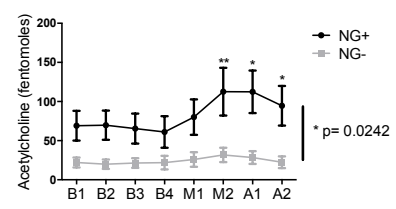

7 Months

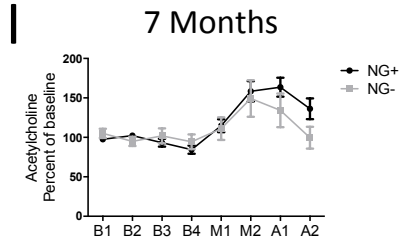


bioRxiv preprint doi: https://doi.org/10.1101/311423; this version posted April 30, 2018. The copyright holder for this preprint (which was not certified by peer review) is the author/funder, who has granted bioRxiv a license to display the preprint in perpetuity. It is made available under aCC-BY-NC-ND 4.0 International license.

A

X-ray
5 Months

B

5 Months
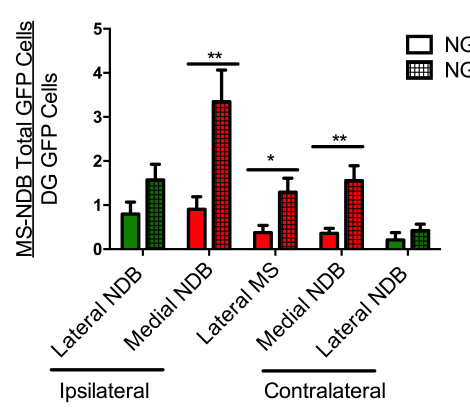

C

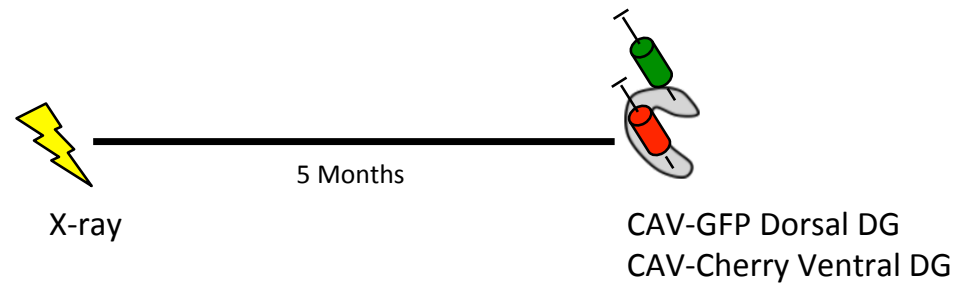

2 Months
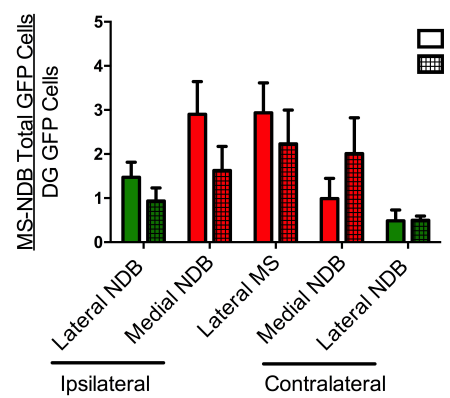

D

NG+

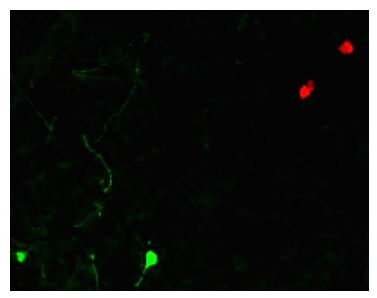

E

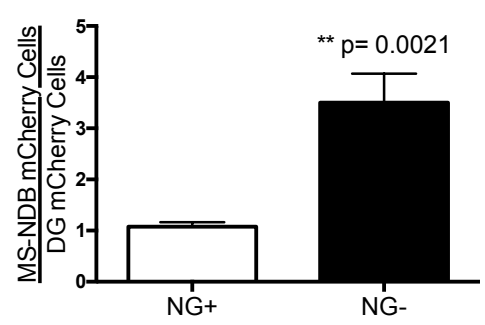

F

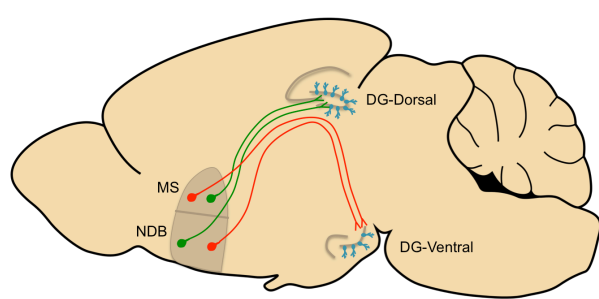

NG-

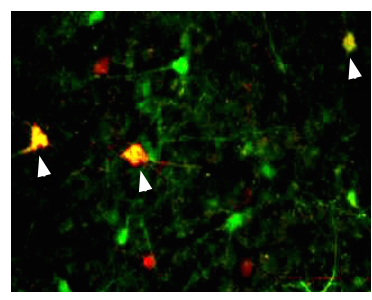

$\square$ NG+

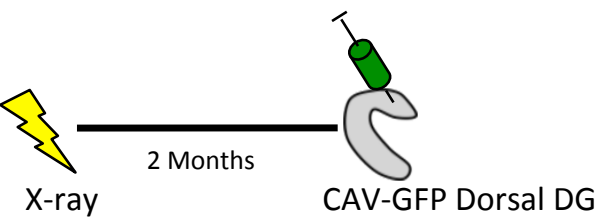

